\title{
Reduced OoB Emission and PAPR Using Partial-OSLM technique in 5G UFMC Systems
}

\author{
Sara Salah \\ EEC Department \\ Tanta University, Egypt \\ sarasalah191@gmail.com
}

\author{
Mahmoud Selem \\ EEC Department \\ Tanta University, Egypt \\ m.sleem2009@gmail.com
}

\author{
Hussein Seleem \\ EEC Department \\ Tanta University, Egypt \\ eltaibee@f-eng.tanta.edu.eg
}

\author{
Mahmoud Attia \\ EEC Department \\ Tanta University, Egypt \\ mahmoudahmedattia@yahoo.com
}

\begin{abstract}
Universal-filtered multi-carrier (UFMC) waveform is considered as a potential candidate for next generation wireless systems due to its robustness against inter-carrier interference (ICI) and the low latency required in $5 \mathrm{G}$ systems. In this paper, a pulse shaping approaches in UFMC technique is studied to reduce the spectral leakage into nearby sub-bands. The performance of various types of window functions such as Chebychev, Hamming, Hanning and Blackman with UFMC are compared. This leads to different coefficients and attenuation shapes, that enabled to choose the proper window function. Finally, a new selective mapping (SLM) peak to average power ratio (PAPR) reduction technique is proposed to enhance the UFMC system performance. Results show that the BER performance of UFMC with all windows functions are the same. However, Blackman window function has a higher attenuation for sidebands compared with others.
\end{abstract}

Index Terms-Universal-filtered multi-carrier (UFMC), Orthogonal frequency division multiplexing (OFDM)

\section{INTRODUCTION}

Fifth-generation $(5 \mathrm{G})$ is the latest iteration of cellular technology and become the most important topic for researchers to greatly increase the speed of wireless networks. With $5 \mathrm{G}$, data transmitted over broadband connections together with advanced antenna technology could travel at rates as high as $20 \mathrm{~Gb} / \mathrm{s}$, which support the expansions of wireless applications [1]. The 5G supports many applications such as Internet of things (IoT) and machine type communications (MTC). Moreover, it is supposed to offer latency of $1 \mathrm{~ms}$ or lower for uses that require real-time feedback.

Over the last decade, an orthogonal frequency division multiplexing (OFDM) waveform is used for $4 \mathrm{G}$ standard [2], [3], which solves multi-path channel problem [4]. The OFDM uses guard band and cyclic prefix (CP) in order to avoid intercarrier interference (ICI) and inter-symbol interference (ISI) respectively [5]. However, OFDM suffers from many problems such as low spectrum efficiency, high power consumption and out-of-band (OoB) emission [6]. The 5G solves these problems by using new waveforms such as filter bank multi-carrier (FBMC) and universal filtered multi-carrier (UFMC) [7].

The FBMC system reduces the OoB emission through filtering each sub-carrier individually without using CP. However, this is inappropriate for some applications as it requires higher filter length. On the other hand, UFMC allows to combine both advantage of OFDM, which filters the frequency band completely and that of FBMC, which filters each sub-carrier individually. It divides the complete frequency band into small number of sub-bands, each one consists of a fixed number of sub-carriers. Therefore, UFMC will reduce the filter length encountered in FBMC technique [8], [9].

The standard UFMC uses Dolph-Chebyshev filter for each sub-band. The main advantages are; 1) In the main filter band, there is no attenuation in order to path the valuable signals without any loss. 2) In the stop band, the attenuation is decaying fast and the filter has very limited transition band in order to avoid OoB emission. 3) With higher filter orders, a good performance can be achieved on the expense of computational cost. Therefore, the trade-off between the performance and complexity need to be optimized [10], [11].

Another problem at UFMC system is the high value of peak to average power ratio (PAPR). This affects the performance of the high power amplifiers (HPAs) in UFMC transmitter. Several techniques have been used to reduce the value of PAPR, such as clipping, precoding, companding, and selective mapping (SLM) techniques. Companding technique can be used in the UFMC system because it is an easy and less complex PAPR reduction [12]. Mu-Law companding technique is based on compressing the large signals and expanding the small signals to protect the small signals from noise. On the other hand, SLM technique is based on generating a set of candidate data blocks by the transmitter. Each data block has the same information of the original data block. The technique selects the block which has the least PAPR for transmission [12]-[14].

This article, initially compares the performance of both UFMC and OFDM systems concerning the bit error rate (BER) in the presence of additive white Gaussian noise (AWGN) and Rayleigh fading channel. The comparison has been applied for different types of modulation formats such as QPSK, 16QAM and 64QAM. Next, the study of the performance of various window functions with UFMC such as Hanning, Hamming, Chebyshev and Blackman are investigated. This helps in choosing the proper window function to design the filter of the proposed UFMC system. Finally, PAPR reduction techniques such as Mu-law companding and partial-orthogonal SLM (POSLM) techniques are proposed in order to enhance 
the performance of UFMC system. Meanwhile, both UFMC and OFDM systems are compared in terms of PAPR and BER performance.

The organization of the paper is as follows. In Section II, UFMC system model is described. The design functions for various types of filters are explained in Section III. In Section IV, PAPR reduction techniques have been presented. Section V, show the simulation results and the performance of UFMC systems compared with OFDM systems. Finally, the concluding remarks are presented in Section VI.

\section{THE UFMC SYSTEM MODEL}

The UFMC communication system is based on dividing the whole $K$ frequency band into $B$ sub-bands. Each sub-band has a fixed number of sub-carriers $m_{l}$, such as $K=B m_{l} \Delta f$, where $\Delta f$ is the sub-carrier band. The UFMC transceiver block diagram is shown in Fig. 1.

The user data bits associated with $i^{\text {th }}$ sub-band are firstly mapped into a column vector $\mathbf{s}_{i} \in \mathbb{C}^{m_{l} \times 1}$ of $m_{l}$ complex data symbols using any digital modulation scheme (e.g., QPSK, 16QAM or 64QAM). The $\mathbf{s}_{i}$ complex data symbols are then passed through $N_{F F T}$-points Inverse Fast Fourier Transform (IFFT) to be converted into time domain. The IFFT output of each sub-band is filtered using digital filter of length $N_{f}$. The output signal can be expressed as:

$$
\mathbf{x}=\sum_{i=1}^{B} \mathbf{x}_{i}=\sum_{i=1}^{B} \mathbf{F}_{i} \mathbf{V}_{i} \mathbf{s}_{i}
$$

where $i=1,2, \ldots, B$ is the index of different sub-bands, $\mathbf{F}_{i} \in$ $\mathbb{C}^{\left(N_{F F T}+N_{f}-1\right) \times N_{F F T}}$ is a Toeplitz matrix composed of the filter impulse response, $\mathbf{V}_{i} \in \mathbb{C}^{N_{F F T} \times m_{l}}$ is the IFFT matrix, and $\mathbf{x}_{i} \in \mathbb{C}^{\left(N_{F F T}+N_{f}-1\right)}$ is the output of each branch. The transmuted signal $\mathbf{x} \in \mathbb{C}^{\left(N_{F F T}+N_{f}-1\right)}$ is the summing of all sub-bands outputs.

The received signal at the receiver can be expressed as,

$$
\mathbf{y}=\mathbf{H} \mathbf{x}+\mathbf{n}
$$

Where $\mathbf{H} \in \mathbb{C}^{\left(N_{F F T}+N_{f}-1\right) \times\left(N_{F F T}+N_{f}-1\right)}$ is the channel convolution matrix that follows the Rayleigh fading distribution and $\mathbf{n} \in \mathbb{C}^{\left(N_{F F T}+N_{f}-1\right) \times 1}$ is a zero mean complex additive white Gaussian noise (AWGN) with variance $\sigma^{2}$.

In UFMC receiver, $2 N_{F F T}$-point Fast Fourier Transform is used to convert the received signal from time domain to frequency domain. Also, zero padding is utilized to avoid inter symbol interference (ISI). The output of FFT passes through frequency domain equalization to detect the transmitted data, $\mathbf{y}_{e q}$. The symbol de-mapping demodulates the output signal from the equalized signal to restore the estimated original data. The estimated original data can be expressed as,

$$
\hat{\mathbf{s}}=\mathbf{U}_{e q}
$$

where, $\mathbf{U} \in \mathbb{C}^{K \times\left(N_{F F T}+N_{f}-1\right)}$ is the UFMC demodulation matrix, and $\mathbf{y}_{e q}$ represents the equalized signal.

\section{FILTER DESIGN}

In this section, different kinds of digital FIR filters will be used. They include Hamming, Hanning, Chebyshev and Blackman filters. Digital FIR filers are used to solve OoB emission problem and to improve PSD efficiency. The characteristics of various filters [15], [16], are summarized as follows.

\section{A. Hamming Window}

$$
w_{\text {hamm }}(n)=\left[0.54-0.46 \cos \left(\frac{2 \pi n}{N-1}\right)\right] R_{N}(n)
$$

where $N$ is the Hamming window length.

The Hamming filter is given as:

$$
f_{\text {hamm }}(n)=f_{d}(n) \cdot w_{\text {hamm }}(n)
$$

where $f_{d}(n)$ is the ideal linear phase filter.

\section{B. Hanning Window}

$$
w_{\text {hann }}(n)=\left[0.5-0.5 \cos \left(\frac{2 \pi n}{N-1}\right)\right] R_{N}(n)
$$

where $R_{N}(n)$ is the rectangular sequence and $N$ is the Hanning window length.

The Hanning filter will be given as:

$$
f_{\text {hann }}(n)=f_{d}(n) \cdot w_{\text {hann }}(n)
$$

\section{Chebyshev Window}

$w_{\text {cheby }}(n)=(-1)^{n} \frac{\cos \left[N \cos ^{-1}[\beta \cos (\pi n / N)]\right]}{\cosh \left[N \cosh ^{-1}(\beta)\right]}, 0 \leq n \leq N-1$

where $N$ is the Chebyshev window length and $\beta$ is the side lobe attenuation.

Therefore, the Dolph-Chebyshev filter will be given as:

$$
f_{\text {cheby }}(n)=f_{d}(n) \cdot w_{\text {cheby }}(n)
$$

\section{Blackman Window}

$w_{\text {black }}(n)=\left[0.42-0.5 \cos \left(\frac{2 \pi n}{N-1}\right)+0.08 \cos \left(\frac{4 \pi n}{N-1}\right)\right] R_{N}(n)$

where $N$ is the Blackman window length and Blackman filter will be written as:

$$
f_{\text {black }}(n)=f_{d}(n) \cdot w_{\text {black }}(n)
$$




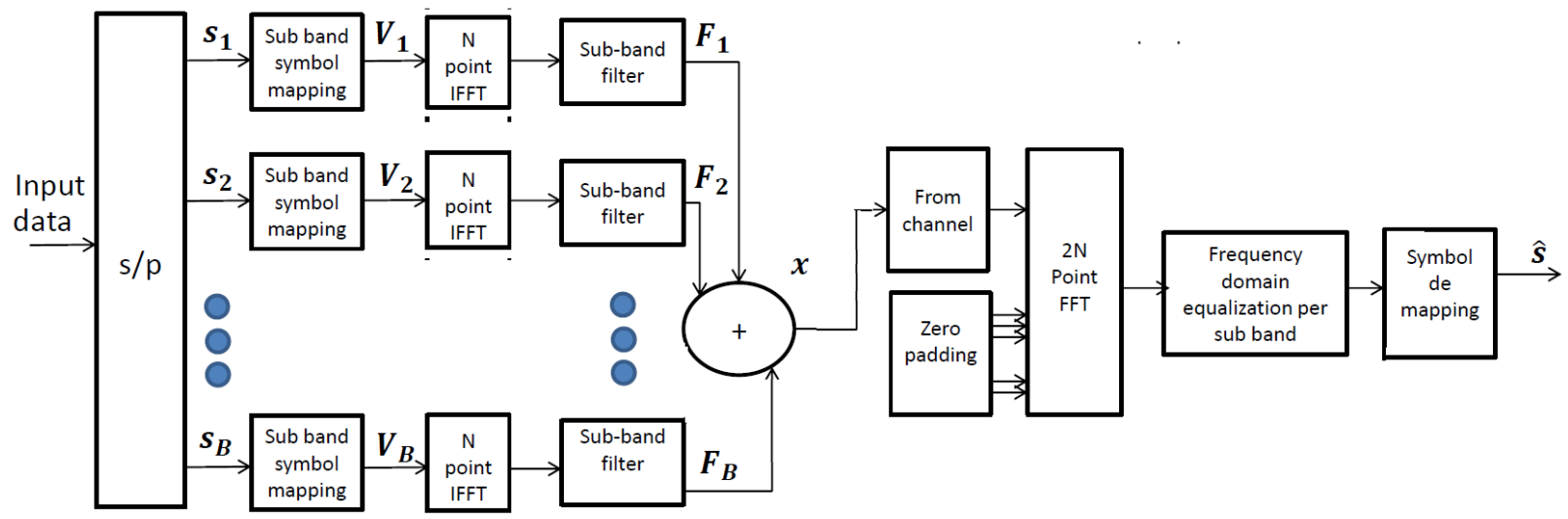

Fig. 1. The UFMC system model

\section{PAPR OF UFMC SYSTEM}

The peak-to-average power ratio (PAPR) will be calculated as the ratio between the maximum power of the samples in a given transmit symbol and the average power of that symbol. High PAPR occurs in a multi-carrier (MC) systems because there are a large number of independently modulated subcarriers. When the different sub-carriers added up coherently, they give a large peak value as compared to average value of the symbol. Consequently, The PAPR will be given as:

$$
P A P R=\frac{\max \left(|x(n)|^{2}\right)}{E\left[|x(n)|^{2}\right]}
$$

where $x(n)$ is the multi-carrier symbols, $\max ()$ denotes the maximum signal power, and $E$ denotes the average signal power.

The high value of PAPR degrades the performance of UFMC system because it causes saturation of the high power amplifier which produces inter modulation product. In the following sections, Mu-Law companding and partial-orthogonal SLM PAPR reduction techniques have been used to reduce PAPR and to increase the efficiency of UFMC system. The complementary cumulative distribution function (CCDF) is used to analyze the efficiency of a PAPR reduction technique.

\section{A. Mu-Law Companding Technique}

The Mu-Law companding offers very good PAPR reduction. It compresses the original UFMC signal at the transmitter end by using the monotonically increasing functions. The signal can be easily recovered at the receiver end by using the inverse transform function before the signal passes through any other operation. Mu-Law companding can be expressed as in [12].

$$
C(x[n])=\operatorname{sgn}(x[n]) \frac{\ln (1+\mu x[n])}{\ln (1+\mu)}
$$

where $\operatorname{sgn}()$ represents the sign function and it is used to maintain the phase of UFMC signal. The $\mu$ parameter represents the compression ratio. If $\mu$ increases, the compression of the amplitude of the transmitted signal increases and offers high PAPR reduction. The inverse companding operation can be expressed as [12]

$$
C^{-1}(y[n])=\operatorname{sgn}(y[n]) \frac{1}{\mu}\left((1+\mu)^{|y[n]|}-1\right)
$$

\section{B. Partial Orthogonal SLM (POSLM) technique}

The selective mapping (SLM) technique is based on generating $U$ candidate vectors of length $N$ identical to the UFMC symbol length. The $U$ vectors is represented by a concatenation of two sub-vectors. The first sub-vector is an orthogonal vector of length $N-\beta$ formed by Hadamard matrix. The second of length $\beta=N / \epsilon$ is formed by random elements taken from the normal distribution, where $\epsilon$ is an integer number greater than one. If $\beta$ increases the random part will increase and the orthogonal part will decease. At the transmitter side, the complex data symbols $s_{i}$ will be multiplied by $U$ phase vectors and the vector with the lowest PAPR is selected for transmission. The transmitted signal can be represented by:

$$
\mathbf{x}^{v}=\sum_{i=1}^{B} F_{i} V_{i} S_{i}^{v}=\left[x_{1}^{v}, x_{2}^{v}, \ldots, x_{N_{F F T}+N_{f}-1}^{v}\right]
$$

where $v$ denotes the signal with the lowest PAPR from the set $U$ and $\mathbf{p}^{v} \in\left\{\mathbf{p}^{u}, 1 \leq u \leq U\right\}$ is the best phase sequence from the set of $U$ vectors. The phase vector $\mathbf{p}^{v}$ can be expressed by:

$$
\mathbf{p}_{k}^{v}=\left[p_{1}^{v}, p_{2}^{v}, \ldots, p_{N_{F F T}+N_{f}-1}^{v}\right]
$$

The reduction of PAPR depends on the number of $U$ phase vectors and the design of the phase rotation factors $p_{k}^{u}=e^{j \phi_{k}^{u}}$ where $\phi_{k}^{u}$ is a random discrete value in the range of 0 to $2 \pi$. At the UFMC receiver side, the transmitted phase vector $\mathbf{p}^{v}$ 
TABLE I

THE UFMC AND OFDM SYSTEM PARAMETERS.

\begin{tabular}{ccc}
\hline Parameter & UFMC & OFDM \\
\hline Number of subcarriers & 300 & 300 \\
\hline IFFT/FFT size & 512 & 512 \\
\hline Modulation order & QPSK, 16QAM,64QAM \\
\hline CP length & - & 36 \\
\hline Filter length & 37 & - \\
\hline Number of sub-bands, $B$ & 30 & - \\
\hline Sub-band size & 10 & -
\end{tabular}

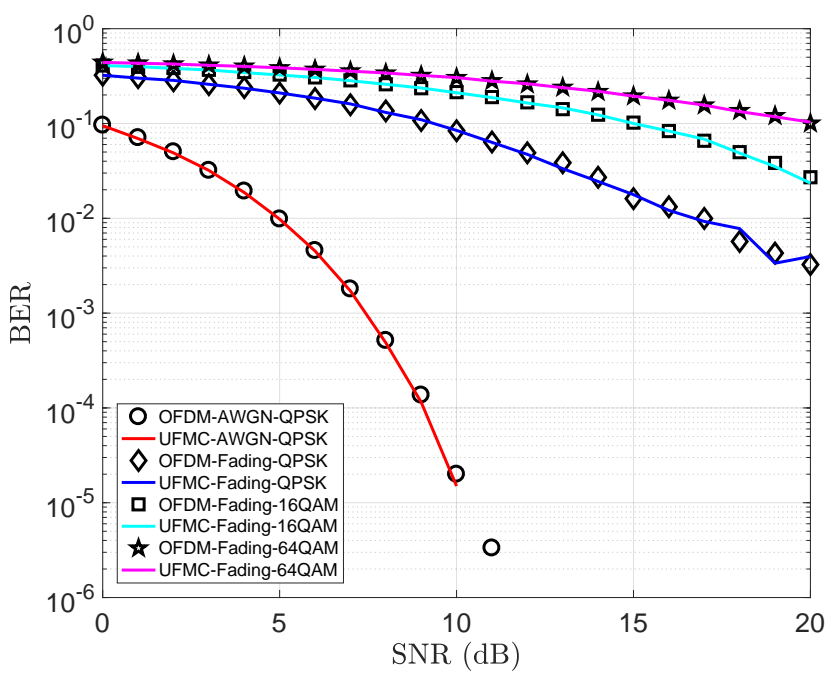

Fig. 2. BER of UFMC using Dolph-Chebyshev filter compared to OFDM.

must be estimated to redirect the data block to its original phases by solving the following optimization problem:

$$
\mathbf{p}^{v}=\underset{u \in\{1,2, \ldots U\}}{\arg \max } \sum_{n=1}^{N_{F F T}+N_{f}-1}\left(\left|y_{n}\right|^{2}, p_{n}^{u}\right)
$$

\section{SimUlation AND ANALYSiS}

The simulation parameters of both UFMC and OFDM are listed as in Table I.

The performance of UFMC and OFDM systems are compared in terms of BER versus signal to noise ratio (SNR). Additionally, the performance of different kind of digital FIR filters in the case of UFMC system is explained, in terms of power spectral density (PSD) efficiency and PAPR.

Figure 2 shows the performance of both UFMC and OFDM systems in the presence of additive white Gaussian noise (AWGN) and Rayleigh fading channels while using different modulation techniques such as QPSK, 16QAM, and 64QAM. The BER performance of both systems are almost the same.

For example, at BER of $10^{-2}$, the SNR of UFMC sytem is the same as SNR for OFDM which is $5 \mathrm{~dB}$ for QPSK modulation technique. The BER performance of UFMC and

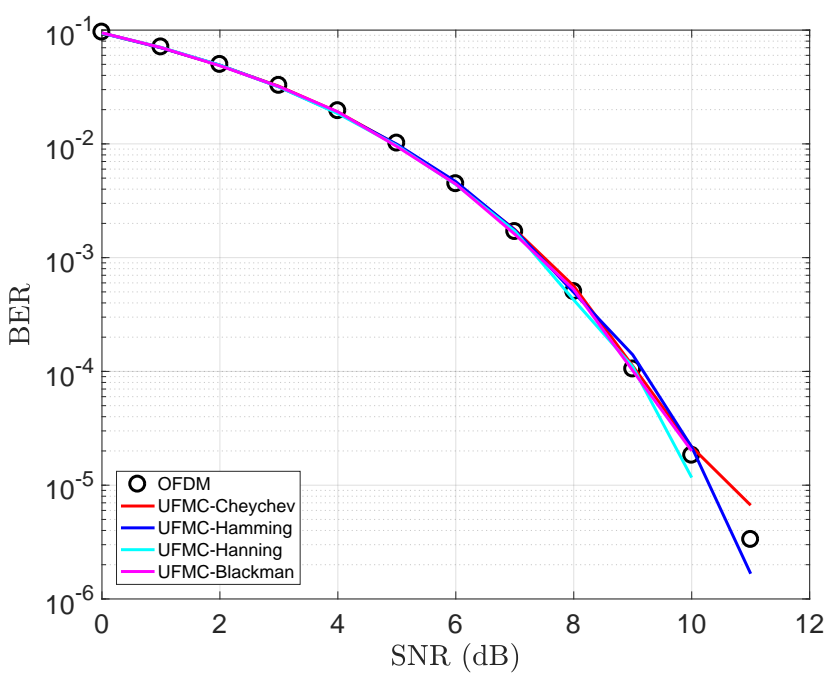

Fig. 3. BER of UFMC for all window functions in QPSK.

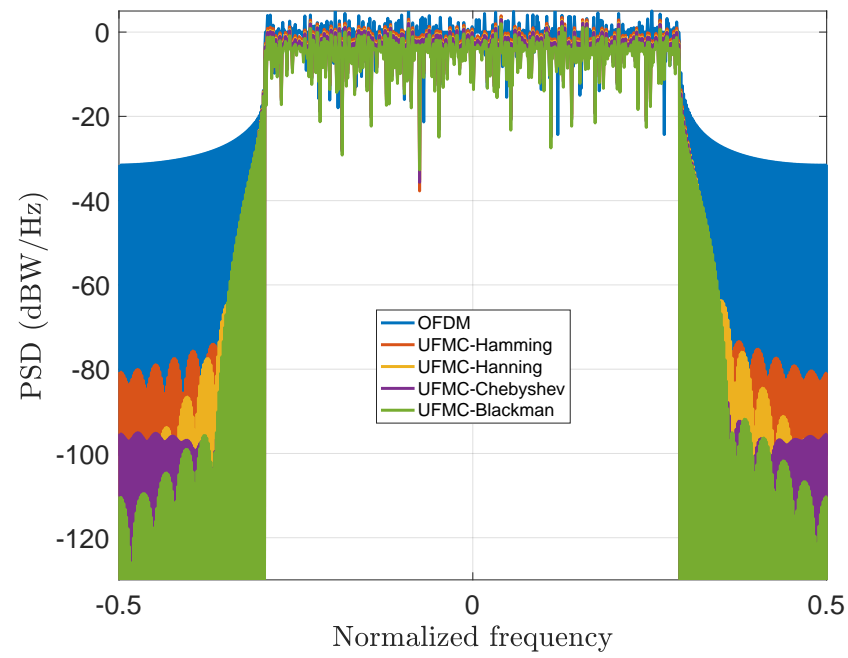

Fig. 4. PSDs of UFMC using different window functions compared to OFDM using QPSK modulation format.

OFDM are degraded in the presence of Rayleigh fading channel. For example, at BER $10^{-2}$ the SNR of UFMC and OFDM are $16 \mathrm{~dB}$ for QPSK. In addition for higher order modulation techniques such as 16QAM and 64QAM, the BER will be degraded again to higher values. For example, at BER of $10^{-1}$, the SNR of UFMC and OFDM are $10 \mathrm{~dB}$ for QPSK and $15 \mathrm{~dB}$ for 16QAM.

On using different types of digital FIR filters instead of Dolph-Chebyshev filter, the BER performance of UFMC is indicated in Fig.3. Although, it can be seen from this figure that the BER performance of UFMC with various filters are similar, they all have a very low OoB emission compared to OFDM as indicated in Fig. 4. It illustrates the different power spectral density (PSD) of UFMC with various window 
TABLE II

THE PAPR FOR BOTH UFMC AND OFDM SYSTEMS.

\begin{tabular}{rcc}
\hline Filter & PAPR(OFDM) & PAPR(UFMC) \\
\hline- & 8.2437 & \\
Hamming & - & 8.5798 \\
Hanning & - & 8.5906 \\
Chebyshev & - & 8.5744 \\
Blackman & - & 8.5714 \\
\hline
\end{tabular}

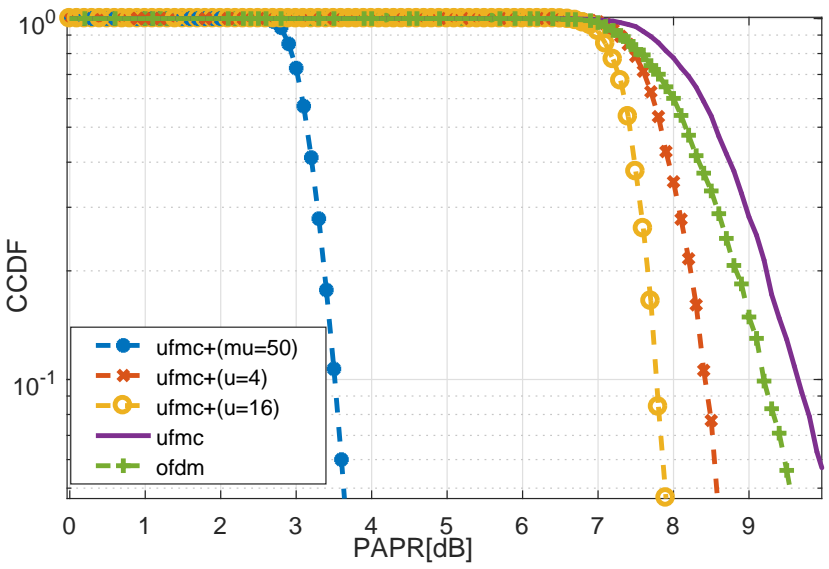

Fig. 5. Comparison of the PAPR of the Mu-Law companding and SLM techniques with the UFMC system using the 16-QAM modulation technique.

functions compared to OFDM. Both Hamming and Hanning windows achieve 50 and $60 \mathrm{~dB}$ respectively lower than OFDM. The Chebyshev window is slightly better than Hanning window. Furthermore, the Blackman window achieves 80dB lower than OFDM. So, the Hamming window is the worst type whereas the Blackman is the best type of these window functions. The calculated peak-to-average power ratio (PAPR) for UFMC system with various filters is tabulated in Table II. Unfortunately, it shows that the PAPR for OFDM is lower than UFMC disregarding the filter type used. Henceforth, different types of PAPR reduction techniques such as $\mathrm{Mu}$ Law companding and partial orthogonal selective mapping (POSLM) techniques are ustilized with UFMC system for both QPSK and 16-QAM modulation. Figure 5 shows the complementary cumulative distribution function (CCDF) of UFMC system with two lengths of the candidate vectors $U$ (i.e., $u=4$ and $u=16$, respectively).

On the other hand, with Mu-Law companding technique $(\mu)$ is set to 50 with 16-QAM modulation technique. Fig. 5 shows that, the value of the PAPR is decreased by using POSLM technique with different values of $(u=4, u=16)$ but, the CCDF curve at $u=16$ is better than the CCDF curve of $u=4$. It should be noted that, the CCDF curve is improved when the value of the candidate vector $U$ is increased. In addition, the CCDF curve for the Mu-Law companding technique with $(\mu=50)$ is more better than the CCDF curves for the POSLM technique $(u=4, u=16)$.

As shown in Fig. 6, however, Mu-Law companding is better

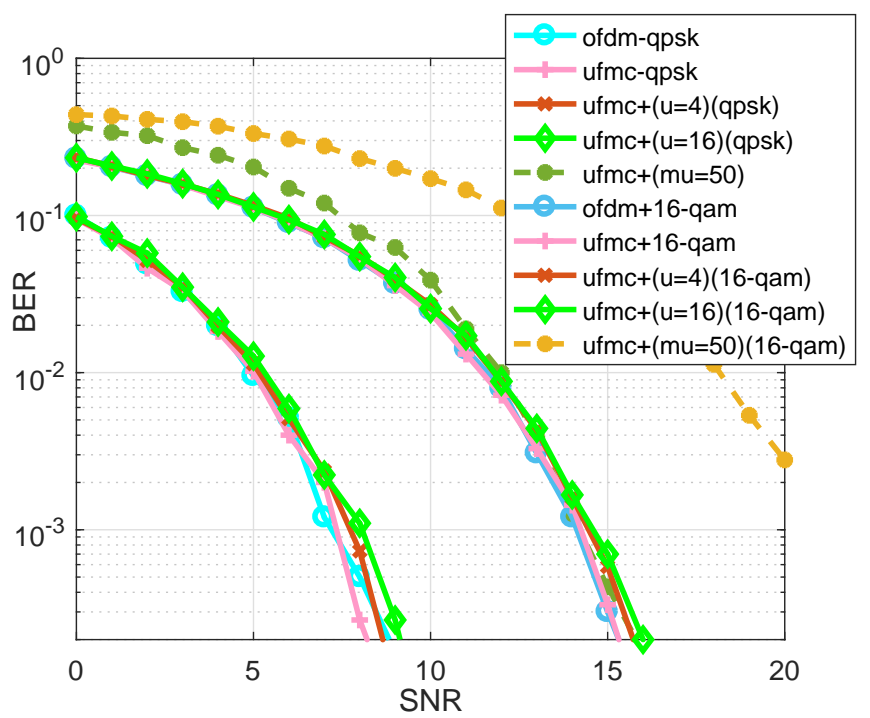

Fig. 6. Comparison of the BER of the Mu-Law companding and SLM techniques with the UFMC system using the QPSK and 16-QAM modulation technique.

than POSLM in terms of CCDF, its BER performance is very poor.

Figure 6 shows the BER performance of the UFMC system by using POSLM technique with different values of the candidate vectors $U$ such as $(u=4, u=16)$. in addition to, using Mu-Law companding technique with $(\mu=50)$ for QAM and 16-QAM modulation techniques. It can be seen that, the POSLM technique has small effect on the BER performance of the UFMC system compared to the standard UFMC system for QAM and 16-QAM modulation techniques. on the other hand, using different values of candidate vectors $U$ have no effect on the BER performance of the POSLM technique for QAM and 16-QAM modulation techniques. Also, the BER of the Mu-Law companding technique is increased for QAM and 16-QAM modulation techniques, because the Mu-Law companding enlarges the small signals in the transmitter which needs expansion in the receiver part of the UFMC system that makes the noise increases.

\section{CONCLUSiON}

The performance of both UFMC and OFDM systems have been investigated in the presence of AWGN and Rayleigh fading channels. A comparison has been applied for different modulation techniques, such as QPSK, 16QAM and 64QAM. Various window functions, such as Chebyshev, Hamming, Hanning and Blackman with UFMC have been studied and lead to choose the Blackman as the proper window in designing the filter, from the PSD point of view. Furthermore, the proposed partial orthogonal SLM PAPR reduction technique have enhanced UFMC sytem performance. It has been prevented the saturation in the high power amplifier that produces inter modulation product and degrades the system performance. 


\section{REFERENCES}

[1] F.-L. Luo and C. Zhang, Signal processing for 5G: algorithms and implementations. John Wiley \& Sons, 2016.

[2] Y. S. Cho, J. Kim, W. Y. Yang, and C. G. Kang, MIMO-OFDM wireless communications with MATLAB. John Wiley \& Sons, 2010.

[3] L. Dai, Z. Wang, and Z. Yang, "Time-frequency training ofdm with high spectral efficiency and reliable performance in high speed environments," IEEE Journal on Selected Areas in Communications, vol. 30, no. 4, pp. 695-707, 2012.

[4] S. Ghorpade and S. Sankpal, "Behaviour of ofdm system using matlab simulation," International Journal of Advanced Computer Research, vol. 3, no. 2 , p. $67,2013$.

[5] T. Hwang, C. Yang, G. Wu, S. Li, and G. Y. Li, "Ofdm and its wireless applications: A survey," IEEE transactions on Vehicular Technology, vol. 58, no. 4, pp. 1673-1694, 2008.

[6] J. Li, K. Kearney, E. Bala, and R. Yang, "A resource block based filtered ofdm scheme and performance comparison," in ICT 2013. IEEE, 2013, pp. 1-5.

[7] Y. Tao, L. Liu, S. Liu, and Z. Zhang, "A survey: Several technologies of non-orthogonal transmission for 5g," China communications, vol. 12, no. 10, pp. 1-15, 2015.

[8] P. N. Rani and C. S. Rani, "Ufmc: The 5g modulation technique," in 2016 IEEE International Conference on Computational Intelligence and Computing Research (ICCIC). IEEE, 2016, pp. 1-3.

[9] R. T. Kamurthi et al., "Review of ufmc technique in 5g," in 2018 International Conference on Intelligent Circuits and Systems (ICICS). IEEE, 2018, pp. 115-120.

[10] X. Cheng, Y. He, B. Ge, and C. He, "A filtered ofdm using fir filter based on window function method," in 2016 IEEE 83rd Vehicular Technology Conference (VTC Spring). IEEE, 2016, pp. 1-5.

[11] K. C. Hu and A. G. Armada, "Sinr analysis of ofdm and f-ofdm for machine type communications," in 2016 IEEE 27th Annual International Symposium on Personal, Indoor, and Mobile Radio Communications (PIMRC). IEEE, 2016, pp. 1-6.

[12] A. F. Almutairi, M. Al-Gharabally, and A. Krishna, "Performance analysis of hybrid peak to average power ratio reduction techniques in 5g ufmc systems," IEEE Access, vol. 7, pp. 80651-80660, 2019.

[13] S. A. Fathy, M. N. Ibrahim, S. S. Elagooz, and H. M. El-Hennawy, "Efficient slm technique for papr reduction in ufmc systems," in 2019 36th National Radio Science Conference (NRSC). IEEE, 2019, pp. 118-125.

[14] M. Al-Rayif, H. Seleem, A. Ragheb, and S. Alshebeili, "Experimental demonstration for papr reduction in ofdm system using partial-oslm technique," Journal of Circuits, Systems and Computers, vol. 27, no. 07, p. 1850106, 2018.

[15] A. V. Oppenheim, Discrete-time signal processing. Pearson Education India, 1999.

[16] F. J. Harris, "On the use of windows for harmonic analysis with the discrete fourier transform," Proceedings of the IEEE, vol. 66, no. 1, pp.5183, Jan. 1978. 